\title{
Designing to Digital Wireless Specifications Using Circuit Envelope Simulation
}

\author{
How-Siang Yap, HP EEsof Division, Hewlett-Packard
}

\begin{abstract}
Circuit Envelope is a circuit simulation technology developed specifically to simulate modern wireless circuits with complex digitally modulated RF signals such as CDMA and TDMA. In this paper, Circuit Envelope technology is explained and is contrasted with Spice and harmonic balance simulators. Applications of Circuit Envelope are then demonstrated in the simulation of phase-locked loops (PLL); amplifier adjacent channel power ratio (ACPR) and noise power ratio (NPR); and IQmodulator error vector magnitude (EVM).
\end{abstract}

\section{Introduction}

Modern communication signals employ sophisticated digital modulations such as $\frac{\pi}{4}$ DQPSK, GMSK and QAM on the RF carrier to improve spectral efficiency. Along with these modulation schemes are new digital wireless standards such as IS-54, GSM and JDC whose specifications are based on the allowable impairments to the digitally modulated RF carriers.

Specifications such as ACPR, EVM and NPR are not correlated to traditional third order intercept (IP3) or gain compression data obtained by stimulating RF components with sinusoidal waveforms. Realistic digitally modulated RF sources are required instead. However, circuit simulation involving such signals with traditional Spice or harmonic balance simulators can be impracticably long or memory-intensive.

This paper starts with a brief review of the characteristics of typical digital wireless communication signals and the difficulty they present to existing time- or frequency-domain circuit simulation technologies. A new circuit simulation technology called Circuit Envelope is then introduced, followed by application examples that has been successfully exploited in the consumer wireless industry.

\section{Characteristics of Consumer Wireless Signals}

RF signals employed in consumer wireless applications [1] occupy a narrow bandwidth relative to its RF carrier. For example a typical cellular phone

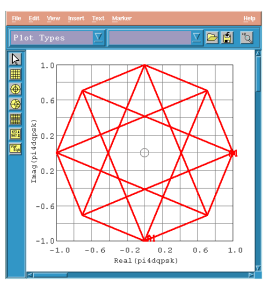

Fig, $1 \frac{\pi}{4}$ DQPSK modulation state diagram signal has a $30 \mathrm{kHz}$ modulation bandwidth on a $1-2 \mathrm{GHz}$ carrier. Digital modulation, in the form of baseband I (in-phase) and Q (quadrature-phase) components, is applied to the RF carrier through an IQ modulator. Fig. 1 shows an unfiltered $\frac{\pi}{4}$ DQPSK modulation state diagram as employed in the North American Digital Cellular (NADC) system [2]. When a digitally modulated narrow band signal passes through the nonlinear components of a communication signal path, the output spectrum consists of narrow-band modulation frequencies around the fundamental and harmonics of the RF carrier.

\section{Spice and Harmonic Balance Limitations}

Spice is a well-known general purpose timedomain circuit simulator. For sufficient practical accuracy, it must sample the highest harmonic frequency of interest by 5-6 times the Nyquist rate. These small time sampling steps must then be simulated over sufficient number of symbols in a digital modulation if we want to observe the modulation spectrum around the fundamental to analyze adjacent channel interference. Consider an NADC signal containing an $890 \mathrm{MHz}$ carrier with a significant third harmonic, and modulated at the symbol rate of $24.3 \mathrm{kHz}$. It requires over 300 million time steps of 62.4 ps to simulate 512 symbols. These 300 million time points must then be FFT'ed to obtain the spectral plot -- another time consuming step.

Harmonic balance [3] is a well-accepted frequency-domain nonlinear circuit simulator. It directly computes the steady-state response of a circuit by calculating the Fourier coefficients of the output solution. Examples include the responses of amplifiers or mixers when stimulated with one or two-tone sinusoidal sources. It is very efficient and accurate when the periodic solution can be represented by of the sum of relatively few steadystate sinusoids or tones: 


$$
v(t)=\sum_{k=0}^{N} V_{k} e^{j \omega_{k} t}
$$

A practical limit for today's computer is $\mathrm{N}<256$ tones for a medium sized circuit. However, this may not adequately represent the continuous spectrum of a transient signal or a non-periodic digitally modulated waveform.

\section{Circuit Envelope Simulation}

Circuit Envelope [4,5] simulation technique addresses the above limitations by applying timedomain techniques on top of the frequency-domain harmonic balance solution. It accepts the input stimulus as RF carriers with time-varying complex envelopes (i.e., amplitude and phase modulations). The output solution is represented as a sum of the RF carriers and their harmonics, each with a timevarying complex envelope:

$$
v(t)=\operatorname{real}\left(\sum_{k=0}^{N} V_{k}(t) e^{j \omega_{k} t}\right)
$$

In contrast to the standard harmonic balance solution, each of the $V_{k}$ terms is no longer a constant representing the steady state value of a discrete tone. Instead, each is now a complex-valued function of time, $V_{k}(t)$ that may represent an arbitrary modulation spectrum around each of the $\omega_{k}$ output harmonics. This spectrum may represent transient signals or pseudo-random digital modulation with their continuous spectrum. Or, it may also include periodic signals with their discrete spectral lines, such as those representing the intermodulation products from a mixer or amplifier under multi-tone sinusoidal excitation.

Circuit Envelope has the fundamental advantage over time-domain simulators in that the time stepsize need only be small enough to capture the bandwidth of the modulation envelope (which is about $30 \mathrm{kHz}$ ), instead of the RF carrier (which is about $1-2 \mathrm{GHz}$ ). Figure 2 pictorially shows the input and output data of the Circuit Envelope simulator.

It should be apparent that the modulation information is completely represented in the time domain as a baseband time-varying complex envelope riding on the RF carriers. This is instead of the multiple separate tones in the harmonic balance system of equations where all frequency components (RF carriers and modulation) must be solved for simultaneously. Typically in Circuit Envelope, the carriers- RF, LO, IF along with their significant

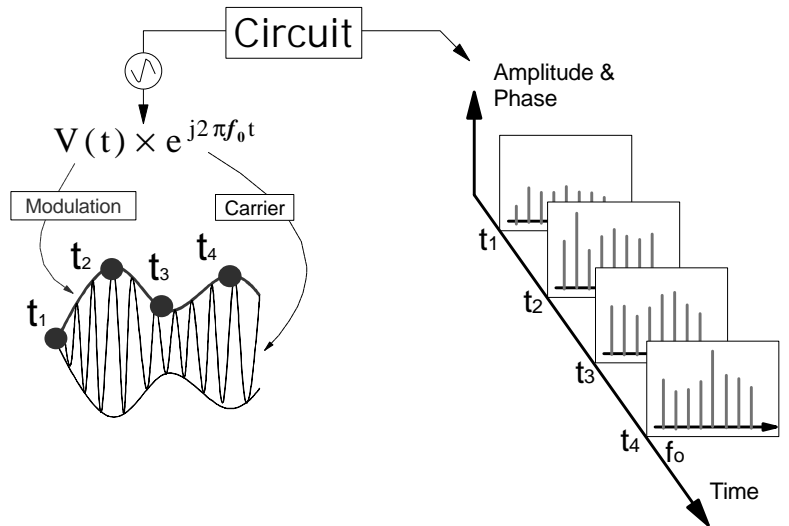

Fig, 2 Circuit Envelope samples the modulation envelope in time and outputs a time-varying spectrum for each envelope time point: $t_{1}, t_{2,} t_{3, \cdots}$

intermods and harmonics are simulated and output as the time-varying spectrum shown in figure 2 . The modulation information is not represented by additional tones in the spectrum, but by the timevarying behavior of the spectrum. Hence, even though Circuit Envelope employs harmonic balance as part of its solution process, the matrix sizes remain reasonable for simulation on even personal computers.

\section{A Simple Example}

Consider a linear capacitor:

$$
\begin{gathered}
\qquad i(t)=C \frac{d v(t)}{d t} \\
\text { where } \quad v(t)=\sum_{k=0}^{N} V_{k}(t) e^{j \omega_{k} t} \\
\text { so } i(t)=\sum_{k=0}^{N}\left(j \omega_{k} C V_{k}(t)+C \frac{d V_{k}(t)}{d t}\right) e^{j \omega_{k} t}
\end{gathered}
$$

The time-varying Fourier coefficients of the currents are:

$$
I_{k}(t)=j \omega_{k} C V_{k}(t)+\frac{d C V_{k}(t)}{d t}
$$

The first term on the RHS is the steady-state term as computed by harmonic balance and it does not depend on past history or derivative information. The second term represents the transient envelope current at frequency $k$ caused by a time varying change in the envelope voltage at frequency $k$. This is what Circuit Envelope has to add on top of the harmonic balance solution during simulation. This derivative term may be computed using the common trapezoidal integration algorithm: 
$\frac{d V_{k}\left(t_{n}\right)}{d t}=\frac{2\left(V_{k}\left(t_{n}\right)-V_{k}\left(t_{n-1}\right)\right)}{\left(t_{n}-t_{n-1}\right)}-\frac{d V_{k}\left(t_{n-1}\right)}{d t}$

Frequency-domain models with arbitrary linear frequency response $H(s)$ can be incorporated into Circuit Envelope using any of the following techniques:

-Impulse response convolution

-Rational polynomial fitting and recursive convolution

-Fixed loss, constant delay model

-Constant around $\omega_{k}$ (no time-domain representation required)

These techniques closely parallel what is done when transient simulators incorporate RF models. However, as opposed to normal transient simulation, the frequency-domain models for Circuit Envelope are evaluated more efficiently and accurately. They only have to represent the frequency response over the relatively narrow modulation bandwidth, and not over the entire range of DC to the maximum harmonic frequency.

\section{Practical Advantages}

Since the output from a Circuit Envelope simulation is a time varying spectrum, we have access to the instantaneous amplitude and phase (or equivalently, the I \& Q) modulation information of each harmonic. An example is shown in figure 3, where the instantaneous amplitude of the fundamental harmonic, $f_{0}$, is plotted versus time. This can represent an oscillator start up, an amplifier pulsed response, or the settling transient of an automatic gain control (AGC) [6]. By similarly examining the higher order harmonics, we can even analyze the instantaneous harmonic content within an

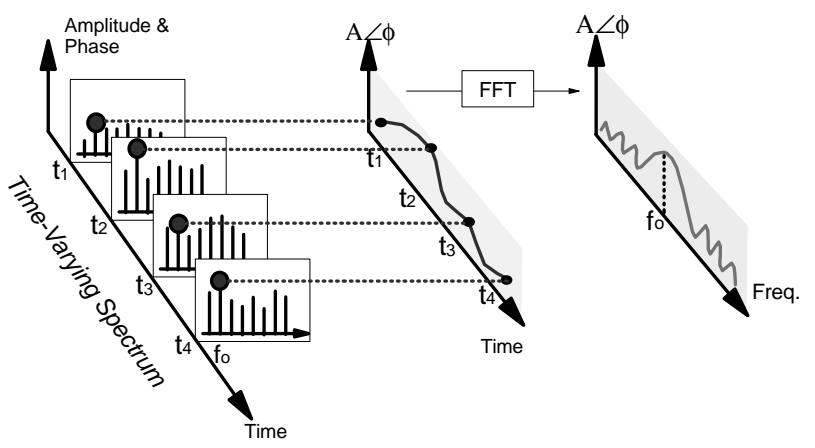

Fig, 3. Instantaneous amplitude and phase (or I \& Q) modulation information of each output harmonic is accessible from the output timevarying spectrum. The modulation spectrum around each harmonic is obtained by performing an FFT on its complex time-varying value.

amplifier pulsed output. The instantaneous frequency of a modulated harmonic can also be derived by differentiating the instantaneous phase with respect to time. This allows us to analyze response involving frequency versus time, such as the frequency settling time of a VCO in a phase-locked loop (PLL) [7] as shown in figure 4.

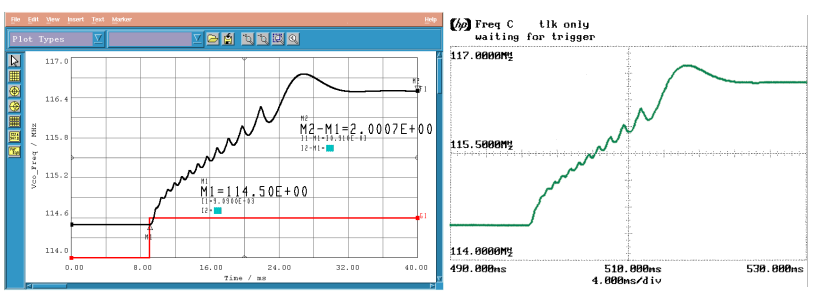

Fig, 4. Simulated vs. measured frequency settling of a PLL versus time

The time-varying amplitude and phase envelope of the RF carrier can also be interpreted as the I and Q components when an arbitrary digital modulation is applied to the RF carrier. Circuit Envelope allows I \& Q baseband digital modulation to be defined by complex numerical expressions or by data files containing I and Q data versus time [8]. Such data may come from a separate digital signal processing (DSP) simulator. The ease of specifying arbitrary digital modulation on the RF carrier makes Circuit Envelope useful for studying the impairments to the digital baseband signals caused by non-ideal RF components $[9,10]$. This is discussed in the next section.

\section{Some Recent Applications}

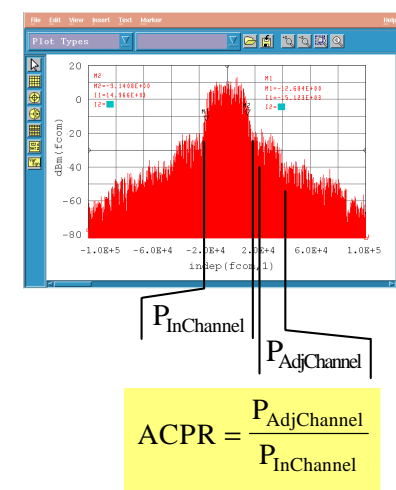

Fig, 5. ACPR measures amount of adjacent channel interference of a digitally modulated RF signal forward. First, select or
define a digital modulation source to modulate the
RF carrier. Then, from the Circuit Envelope output

Adjacent channel power ratio (ACPR) is specified in all wireless standards involving digital modulation. It is a measure of adjacent channel interference caused by spectral regrowth of the digitally modulated RF signal in the amplifier and mixer. Figure 5 shows how ACPR is derived. Simulating in Circuit Envelope is straight- 
time-varying spectrum, Fourier transform the complex time-varying envelope of the desired harmonic (e.g., IF or RF) to obtain the modulation spectrum around that harmonic. Lastly, integrate and ratio the power within the adjacent-channel and inchannel to obtain ACPR. The channel separation and bandwidths to integrate over are specified in the wireless standards.

Noise power ratio (NPR) is a measure of the spurious free dynamic range of a multi-channel amplifier such as a base station, CATV or satellite repeater. Here, multi-channel modulation is applied to the RF carrier. The center channel is notched off (i.e., set to zero). The output will have intermodulation products filling the notched channel. The ratio of the power in the notched channel to an adjacent filled channel is the NPR as shown in figure 6.

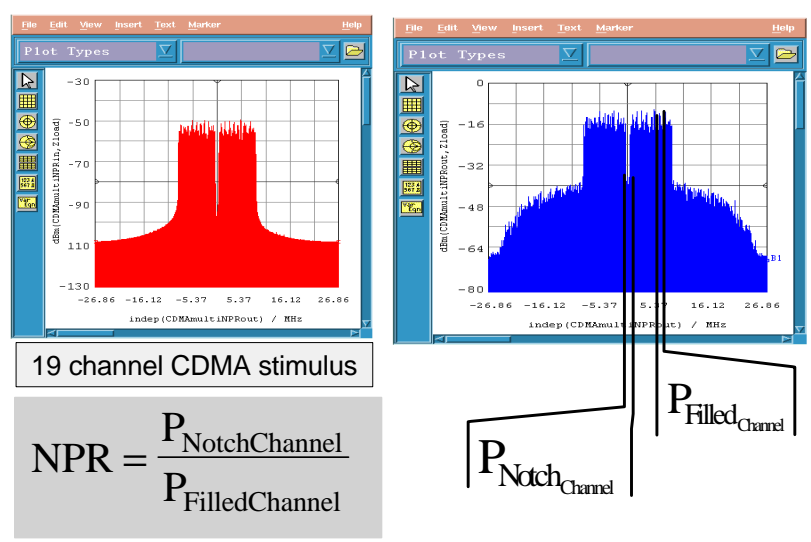

Fig, 6. NPR simulation realistically analyzes the spurious-free dynamic range of multi-channel amplifiers

Another important digital wireless specification is error vector magnitude $(\mathrm{EVM})$. It measures the distortion suffered by the digital modulation constellation in terms of the residual RMS error after compensating for gain, delay, phase rotation and origin shift in the signal path. Figure 7 shows how EVM is computed for a $\frac{\pi}{4}$ DQPSK modulation.

\section{Conclusion}

Circuit Envelope allows circuits with transient or digitally modulated RF signals to be simulated much more efficiently than existing time- and frequencydomain simulators by exploiting the benefits of both techniques. That is, by processing the complex modulation information in the time-domain, while efficiently handling the RF carriers in the frequencydomain.

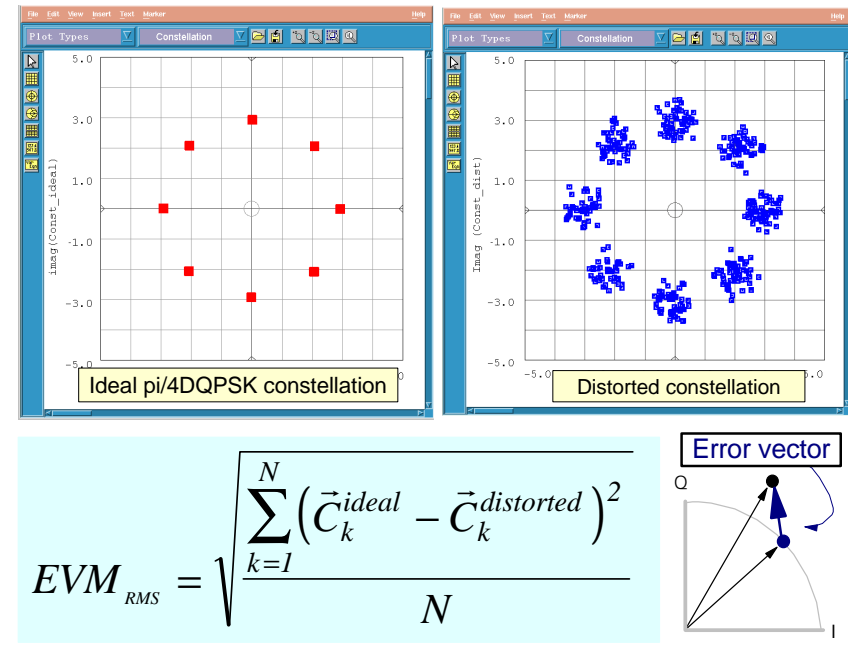

Fig, 7. EVM measures distortion of digital modulation constellation. $\mathrm{N}$ is the number of symbols to sum over and is defined in the wireless standards

\section{Acknowledgments}

I would like to thank David Sharrit and Andy Howard for many insightful discussions on this subject.

\section{References}

[1] Larson, "RF and Microwave Circuit Design for Wireless Communications," Artech House, 1996

[2] Gibson, "The Mobile Communications Handbook," CRC Press, 1996

[3] Kundert, White, \& Sangiovanni-Vincentelli, "SteadyState methods for Simulating Analog and Microwave Circuits," Kluwer Academic Publishers, 1990

[4] HP EEsof MDS Manual, v7.0, "Designer's Task Ref. Vol. 4, Circuit Envelope Simulation," July 1996

[5] Sharrit, "New Method of analysis of Communication Systems," MTTS'96 WMFA: Nonlinear CAD Workshop, June 96

[6] Howard \& Yap, "Circuit Envelope Simulator Technical Seminar," HP EEsof, 1995

[7] Howard, "Software Advances Aid in Simulating PLLBased Circuits," MW \& RF, September 1996.

[8] Pleasant, "HP EEsof Product Note 85150-6, Sharing Data Between the Microwave Design System and OmniSys/CDS," 1995

[9] Yap, "Designing Communications Circuits To New Digital Wireless Standards," Wireless Systems Design, September 1996.

[10] Sevic \& Staudinger, "Simulation of Adjacent-Channel Power for Digital Wireless Communication Systems," Microwave Journal, October 1996 